\title{
Analysis of Caenorhabditis elegans via Bioinformatics Approaches Basis on their Precursors Statistics Values
}

\author{
Alka Dubey ${ }^{1^{*}}$, Shashi Prabha Agarwal ${ }^{2}$, Neelesh Yadav ${ }^{3}$ and Rajnish Kumar ${ }^{2}$ \\ ${ }^{1}$ Bioinformatics Infrastructure Facility (Funded by Dept. of Biotechnology, Govt. of India), Forest Research Institute, Dehradun, India \\ ${ }^{2}$ Assistant Professor, D.A-V College Kanpur, India \\ ${ }^{3}$ Bioinformatics Centre, Forest Research Institute, Dehradun, India
}

*Corresponding author: Alka Dubey, Visiting Research Associate, in Bioinformatics Infrastructure Facility (Funded by Dept. of Biotechnology, Govt. of India) of Forest Research Institute, Dehradun, India, Tel: +08394837352; E-mail: alkabioinfo964@gmail.com

Rec date: Mar 28, 2016; Acc date: June 9, 2016; Pub date: June 13, 2016

Copyright: (C) 2016 Dubey A, et al. This is an open-access article distributed under the terms of the Creative Commons Attribution License, which permits unrestricted use, distribution, and reproduction in any medium, provided the original author and source are credited.

\begin{abstract}
MicroRNA (miRNA) are a class of small regulatory non coding RNAs. These are about 21 to 25 nucleotides in length. Analysis of miRNA is leading to new paradigms for control of gene expression during plants and animals. Most noncoding RNAs are characterized by a specific secondary structures that determine their function. In present study we determine the minimum free energy (MFE) of $C$. elegans precursor's sequences. That retrieves from mirBASE.
\end{abstract}

Keywords MicroRNA; C. elegans; Minimum free energy (MFE); Noncoding; Transcriptional regulators; Ribonuclease III

\section{Introduction}

Micro RNA regulates gene expression. miRNAs are well conserved in both plants and animals, and are thought to be a vital and evolutionarily ancient component of genetic regulation [1]. Mature microRNAs (miRNAs) are a class of naturally occurring small noncoding RNA molecules; about 21 to 25 nucleotides in length MicroRNAs are partially complementary to one or more messenger RNA (mRNA) molecules and their main function is to down- regulate gene expression in a variety of manners, including translational repression, mRNA cleavage and deadenylation [2]. miRNAs are a class of post-transcriptional regulators [3]. Most non-coding RNAs are characterized by a specific secondary and tertiary structure that determines their function.

Analysis of miRNAs is leading to new paradigms for control of gene expression during development in plants and animals. MiRNAs arise from larger precursor molecules that can fold into a stable stem-loop structure [4-8]. Those structures are processed by ribonuclease III-like nuclease Dicer in animals and Dicer like in plants and all have a typical stem-loop shape [4-11].

In present study we predicted the minimum free energy (MFE) values of secondary structures of noncoding RNA sequences, such as microRNA precursors of $C$. elegans with the help of computational software miRBase.

\section{Methodology}

The precursors (pre-miRNA) sequences of $C$. elegans were retrieved from miRBase and then go for secondary structure with optimal minimum free energy [12]. Optimal minimum free energy was found out with the help of RNA fold web servers (http://rna.tbi.univie.ac.at) then retrieve the sequence of miRNA from miRBase. Present study which is exclusively based on in silico firstly retrieves precursor sequences from miRBase and then retrieve sequence is submitted in RNA fold web server for minimum free energy values and calculated the minimum free energy for objective analysis [13].

\begin{tabular}{|l|l|l|l|l|l|}
\hline S.No. & $\begin{array}{l}\text { Precursor Accession } \\
\text { No. }\end{array}$ & $\begin{array}{l}\text { Free energy of } \\
\text { thermodynamics }\end{array}$ & $\begin{array}{l}\text { Frequency of MFE } \\
\text { structure }\end{array}$ & $\begin{array}{l}\text { Ensenbled } \\
\text { diversity }\end{array}$ & $\begin{array}{l}\text { Minimum free energy of } \\
\text { centeriod } \\
\text { structure }\end{array}$ \\
\hline 1 & $>$ Optimal secondary \\
minimum free energy
\end{tabular}


Citation: Dubey A, Agarwal SP, Yadav N, Kumar R (2016) Analysis of Caenorhabditis elegans via Bioinformatics Approaches Basis on their Precursors Statistics Values . Next Generat Sequenc \& Applic 3: 126. doi:10.4172/2469-9853.1000126

Page 2 of 5

\begin{tabular}{|c|c|c|c|c|c|c|}
\hline 8 & >cel-mir-37 MI0000008 & $-43.70 \mathrm{kcal} / \mathrm{mol}$ & $16.70 \%$ & 3.06 & $-42.60 \mathrm{kcal} / \mathrm{mol}$ & $-42.60 \mathrm{kcal} / \mathrm{mol}$ \\
\hline 9 & >cel-mir-38 MI0000009 & $-49.47 \mathrm{kcal} / \mathrm{mol}$ & $4.06 \%$ & 7.27 & $-47.50 \mathrm{kcal} / \mathrm{mol}$ & $-47.50 \mathrm{kcal} / \mathrm{mol}$ \\
\hline 10 & >cel-mir-39 MI0000010 & $-44.12 \mathrm{kcal} / \mathrm{mol}$ & $16.28 \%$ & 3.45 & $-43.00 \mathrm{kcal} / \mathrm{mol}$ & $-43.00 \mathrm{kcal} / \mathrm{mol}$ \\
\hline 11 & >cel-mir-40 MI0000011 & $-46.35 \mathrm{kcal} / \mathrm{mol}$ & $29.44 \%$ & 2.61 & $-45.60 \mathrm{kcal} / \mathrm{mol}$ & $-45.60 \mathrm{kcal} / \mathrm{mol}$ \\
\hline 12 & >cel-mir-41 MI0000012 & $-41.95 \mathrm{kcal} / \mathrm{mol}$ & $29.48 \%$ & 9.07 & $-41.20 \mathrm{kcal} / \mathrm{mol}$ & $-41.20 \mathrm{kcal} / \mathrm{mol}$ \\
\hline 13 & >cel-mir-42 MI0000013 & $-41.91 \mathrm{kcal} / \mathrm{mol}$ & $14.00 \%$ & 4.63 & $-38.10 \mathrm{kcal} / \mathrm{mol}$ & $-40.70 \mathrm{kcal} / \mathrm{mol}$ \\
\hline 14 & >cel-mir-43 MI0000014 & $-47.24 \mathrm{kcal} / \mathrm{mol}$ & $18.65 \%$ & 4.27 & $-46.20 \mathrm{kcal} / \mathrm{mol}$ & $-46.20 \mathrm{kcal} / \mathrm{mol}$ \\
\hline 15 & >cel-mir-44 MI0000015 & $-44.32 \mathrm{kcal} / \mathrm{mol}$ & $5.18 \%$ & 8.74 & $-38.10 \mathrm{kcal} / \mathrm{mol}$ & $-42.50 \mathrm{kcal} / \mathrm{mol}$ \\
\hline 16 & >cel-mir-45 MI0000016 & $-42.70 \mathrm{kcal} / \mathrm{mol}$ & $37.73 \%$ & 4.26 & $-42.10 \mathrm{kcal} / \mathrm{mol}$ & $-42.10 \mathrm{kcal} / \mathrm{mol}$ \\
\hline 17 & >cel-mir-46 MI0000017 & $-40.52 \mathrm{kcal} / \mathrm{mol}$ & $10.03 \%$ & 12.08 & $-39.10 \mathrm{kcal} / \mathrm{mol}$ & $-39.10 \mathrm{kcal} / \mathrm{mol}$ \\
\hline 18 & >cel-mir-47 MI0000018 & $-42.03 \mathrm{kcal} / \mathrm{mol}$ & $41.98 \%$ & 4.90 & $-41.50 \mathrm{kcal} / \mathrm{mol}$ & $-41.50 \mathrm{kcal} / \mathrm{mol}$ \\
\hline 19 & >cel-mir-48 MI0000019 & $-34.68 \mathrm{kcal} / \mathrm{mol}$ & $14.65 \%$ & 5.36 & $-33.10 \mathrm{kcal} / \mathrm{mol}$ & $-33.50 \mathrm{kcal} / \mathrm{mol}$ \\
\hline 20 & >cel-mir-49 MI0000020 & $-38.97 \mathrm{kcal} / \mathrm{mol}$ & $17.61 \%$ & 4.69 & $-37.70 \mathrm{kcal} / \mathrm{mol}$ & $-37.90 \mathrm{kcal} / \mathrm{mol}$ \\
\hline 21 & >cel-mir-50 MI0000021 & $-61.30 \mathrm{kcal} / \mathrm{mol}$ & $8.75 \%$ & 5.84 & $-59.80 \mathrm{kcal} / \mathrm{mol}$ & $-59.80 \mathrm{kcal} / \mathrm{mol}$ \\
\hline 22 & >cel-mir-51 MI0000022 & $-34.90 \mathrm{kcal} / \mathrm{mol}$ & $0.40 \%$ & 5.53 & $-33.30 \mathrm{kcal} / \mathrm{mol}$ & $-31.50 \mathrm{kcal} / \mathrm{mol}$ \\
\hline 23 & >cel-mir-52 MI0000023 & $-26.80 \mathrm{kcal} / \mathrm{mol}$ & $2.82 \%$ & 7.58 & $-24.50 \mathrm{kcal} / \mathrm{mol}$ & $-24.60 \mathrm{kcal} / \mathrm{mol}$ \\
\hline 24 & >cel-mir-53 MI0000024 & $-31.59 \mathrm{kcal} / \mathrm{mol}$ & $10.46 \%$ & 6.92 & $-30.20 \mathrm{kcal} / \mathrm{mol}$ & $-30.20 \mathrm{kcal} / \mathrm{mol}$ \\
\hline 25 & >cel-mir-54 MI0000025 & $-35.03 \mathrm{kcal} / \mathrm{mol}$ & $6.00 \%$ & 8.56 & $-31.00 \mathrm{kcal} / \mathrm{mol}$ & $-33.30 \mathrm{kcal} /$ \\
\hline 26 & >cel-mir-55 MI0000026 & $-35.63 \mathrm{kcal} / \mathrm{mol}$ & $15.91 \%$ & 7.79 & $-34.50 \mathrm{kcal} / \mathrm{mol}$ & $-34.50 \mathrm{kcal} / \mathrm{mol}$ \\
\hline 27 & >cel-mir-56 MI0000027 & $-42.07 \mathrm{kcal} / \mathrm{mol}$ & $15.09 \%$ & 5.96 & $-38.80 \mathrm{kcal} / \mathrm{mol}$ & $-40.90 \mathrm{kcal} / \mathrm{mol}$ \\
\hline 28 & >cel-mir-57 MI0000028 & $-32.39 \mathrm{kcal} / \mathrm{mol}$ & $27.97 \%$ & 5.52 & $-31.60 \mathrm{kcal} / \mathrm{mol}$ & $-31.60 \mathrm{kcal} / \mathrm{mol}$ \\
\hline 29 & >cel-mir-58a MI0000029 & $-37.29 \mathrm{kcal} / \mathrm{mol}$ & $10.42 \%$ & 8.52 & $-35.60 \mathrm{kcal} / \mathrm{mol}$ & $-35.90 \mathrm{kcal} / \mathrm{mol}$ \\
\hline 30 & >cel-mir-59 MI0000030 & $-36.94 \mathrm{kcal} / \mathrm{mol}$ & $15.65 \%$ & 7.37 & $-33.30 \mathrm{kcal} / \mathrm{mol}$ & $-35.80 \mathrm{kcal} / \mathrm{mol}$ \\
\hline 31 & >cel-mir-60 MI0000031 & $-36.59 \mathrm{kcal} / \mathrm{mol}$ & $3.38 \%$ & 9.31 & $-33.30 \mathrm{kcal} / \mathrm{mol}$ & $-34.50 \mathrm{kcal} / \mathrm{mol}$ \\
\hline 32 & >cel-mir-61 MI0000032 & $-52.19 \mathrm{kcal} / \mathrm{mol}$ & $6.45 \%$ & 5.23 & $-50.50 \mathrm{kcal} / \mathrm{mol}$ & $-50.50 \mathrm{kcal} / \mathrm{mol}$ \\
\hline 33 & >cel-mir-63 MI0000034 & $-38.66 \mathrm{kcal} / \mathrm{mol}$ & $24.84 \%$ & 5.78 & $-37.80 \mathrm{kcal} / \mathrm{mol}$ & $-37.80 \mathrm{kcal} / \mathrm{mol}$ \\
\hline 34 & >cel-mir-64 MI0000035 & $-37.69 \mathrm{kcal} / \mathrm{mol}$ & $6.45 \%$ & 9.76 & $-31.40 \mathrm{kcal} / \mathrm{mol}$ & $-36.00 \mathrm{kcal} / \mathrm{mol}$ \\
\hline 35 & >cel-mir-65 MI0000036 & $-42.68 \mathrm{kcal} / \mathrm{mol}$ & $33.35 \%$ & 2.72 & $-42.00 \mathrm{kcal} / \mathrm{mol}$ & $-42.00 \mathrm{kcal} / \mathrm{mol}$ \\
\hline 36 & >cel-mir-66 MI0000037 & $-39.16 \mathrm{kcal} / \mathrm{mol}$ & $34.08 \%$ & 2.53 & $-38.50 \mathrm{kcal} / \mathrm{mol}$ & $-38.50 \mathrm{kcal} / \mathrm{mol}$ \\
\hline 37 & >cel-mir-67 MI0000038 & $-32.72 \mathrm{kcal} / \mathrm{mol}$ & $8.45 \%$ & 5.96 & $-31.20 \mathrm{kcal} / \mathrm{mol}$ & $-31.20 \mathrm{kcal} / \mathrm{mol}$ \\
\hline 38 & >cel-mir-70 MI0000041 & $-32.88 \mathrm{kcal} / \mathrm{mol}$ & $17.40 \%$ & 5.12 & $-31.10 \mathrm{kcal} / \mathrm{mol}$ & $-31.80 \mathrm{kcal} / \mathrm{mol}$ \\
\hline 39 & >cel-mir-71 MI0000042 & $-37.50 \mathrm{kcal} / \mathrm{mol}$ & $19.59 \%$ & 5.77 & $-36.50 \mathrm{kcal} / \mathrm{mol}$ & $-36.50 \mathrm{kcal} / \mathrm{mol}$ \\
\hline 40 & >cel-mir-72 MI0000043 & $-43.59 \mathrm{kcal} / \mathrm{mol}$ & $16.92 \%$ & 4.07 & $-42.50 \mathrm{kcal} / \mathrm{mol}$ & $-42.50 \mathrm{kcal} / \mathrm{mol}$ \\
\hline 41 & >cel-mir-73 MI0000044 & $-36.16 \mathrm{kcal} / \mathrm{mol}$ & $13.05 \%$ & 6.62 & $-33.80 \mathrm{kcal} / \mathrm{mol}$ & $-34.90 \mathrm{kcal} / \mathrm{mol}$ \\
\hline 42 & >cel-mir-74 MI0000045 & $-38.57 \mathrm{kcal} / \mathrm{mol}$ & $24.51 \%$ & 4.22 & $-37.70 \mathrm{kcal} / \mathrm{mol}$ & $-37.70 \mathrm{kcal} / \mathrm{mol}$ \\
\hline 43 & >cel-mir-75 MI0000046 & $-34.87 \mathrm{kcal} / \mathrm{mol}$ & $7.86 \%$ & 5.79 & $-33.30 \mathrm{kcal} / \mathrm{mol}$ & $-33.30 \mathrm{kcal} / \mathrm{mol}$ \\
\hline
\end{tabular}


Citation: Dubey A, Agarwal SP, Yadav N, Kumar R (2016) Analysis of Caenorhabditis elegans via Bioinformatics Approaches Basis on their Precursors Statistics Values . Next Generat Sequenc \& Applic 3: 126. doi:10.4172/2469-9853.1000126

Page 3 of 5

\begin{tabular}{|c|c|c|c|c|c|c|}
\hline 44 & >cel-mir-77 MI0000048 & $-35.74 \mathrm{kcal} / \mathrm{mol}$ & $6.97 \%$ & 9.80 & $-32.10 \mathrm{kcal} / \mathrm{mol}$ & $-34.10 \mathrm{kcal} / \mathrm{mol}$ \\
\hline 45 & >cel-mir-79 MI0000050 & $-33.50 \mathrm{kcal} / \mathrm{mol}$ & $19.60 \%$ & 4.26 & $-31.10 \mathrm{kcal} / \mathrm{mol}$ & $-32.50 \mathrm{kcal} / \mathrm{mol}$ \\
\hline 46 & >cel-mir-80 MI0000051 & $-29.47 \mathrm{kcal} / \mathrm{mol}$ & $6.64 \%$ & 12.39 & $-27.80 \mathrm{kcal} / \mathrm{mol}$ & $-27.80 \mathrm{kcal} / \mathrm{mol}$ \\
\hline 47 & >cel-mir-81 MI0000052 & $-42.01 \mathrm{kcal} / \mathrm{mol}$ & $5.32 \%$ & 7.80 & $-39.90 \mathrm{kcal} / \mathrm{mol}$ & $-40.20 \mathrm{kcal} / \mathrm{mol}$ \\
\hline 48 & >cel-mir-82 MI0000053 & $-34.58 \mathrm{kcal} / \mathrm{mol}$ & $27.98 \%$ & 2.55 & $-33.80 \mathrm{kcal} / \mathrm{mol}$ & $-33.80 \mathrm{kcal} / \mathrm{mol}$ \\
\hline 49 & >cel-mir-83 MI0000054 & $-28.16 \mathrm{kcal} / \mathrm{mol}$ & $10.94 \%$ & 11.86 & $-22.50 \mathrm{kcal} / \mathrm{mol}$ & $-26.80 \mathrm{kcal} / \mathrm{mol}$ \\
\hline 50 & >cel-mir-84 MI0000055 & $-24.11 \mathrm{kcal} / \mathrm{mol}$ & $14.01 \%$ & 2.71 & $-22.90 \mathrm{kcal} / \mathrm{mol}$ & $-22.90 \mathrm{kcal} / \mathrm{mol}$ \\
\hline 51 & >cel-mir-85 MI0000056 & $-40.70 \mathrm{kcal} / \mathrm{mol}$ & $7.44 \%$ & 6.18 & $-38.70 \mathrm{kcal} / \mathrm{mol}$ & $-39.10 \mathrm{kcal} / \mathrm{mol}$ \\
\hline 52 & >cel-mir-86 MI0000057 & $-43.84 \mathrm{kcal} / \mathrm{mol}$ & $15.83 \%$ & 3.50 & $-40.30 \mathrm{kcal} / \mathrm{mol}$ & $-42.70 \mathrm{kcal} / \mathrm{mol}$ \\
\hline 53 & >cel-mir-87 MI0000058 & $-46.54 \mathrm{kcal} / \mathrm{mol}$ & $1.63 \%$ & 5.79 & $-44.90 \mathrm{kcal} / \mathrm{mol}$ & $-44.00 \mathrm{kcal} / \mathrm{mol}$ \\
\hline 54 & >cel-mir-90 MI0000059 & $-44.62 \mathrm{kcal} / \mathrm{mol}$ & $10.02 \%$ & 4.46 & $-43.20 \mathrm{kcal} / \mathrm{mol}$ & $-43.20 \mathrm{kcal} / \mathrm{mol}$ \\
\hline 55 & >cel-mir-124 MI0000302 & $-39.59 \mathrm{kcal} / \mathrm{mol}$ & $14.58 \%$ & 6.87 & $-36.40 \mathrm{kcal} / \mathrm{mol}$ & $-38.40 \mathrm{kcal} / \mathrm{mol}$ \\
\hline 56 & >cel-mir-228 MI0000303 & $-44.86 \mathrm{kcal} / \mathrm{mol}$ & $15.23 \%$ & 4.06 & $-41.90 \mathrm{kcal} / \mathrm{mol}$ & $-43.70 \mathrm{kcal} / \mathrm{mol}$ \\
\hline 57 & >cel-mir-229 MI0000304 & $-55.67 \mathrm{kcal} / \mathrm{mol}$ & $1.54 \%$ & 17.64 & $-50.90 \mathrm{kcal} / \mathrm{mol}$ & $-53.10 \mathrm{kcal} / \mathrm{mol}$ \\
\hline 58 & >cel-mir-230 MI0000305 & $-39.42 \mathrm{kcal} / \mathrm{mol}$ & $5.19 \%$ & 6.85 & $-37.50 \mathrm{kcal} / \mathrm{mol}$ & $-37.60 \mathrm{kcal} / \mathrm{mol}$ \\
\hline 59 & >cel-mir-231 MI0000306 & $-33.40 \mathrm{kcal} / \mathrm{mol}$ & $5.40 \%$ & 8.80 & $-31.60 \mathrm{kcal} / \mathrm{mol}$ & $-31.60 \mathrm{kcal} / \mathrm{mol}$ \\
\hline 60 & >cel-mir-232 MI0000307 & $-37.20 \mathrm{kcal} / \mathrm{mol}$ & $16.90 \%$ & 5.00 & $-35.80 \mathrm{kcal} / \mathrm{mol}$ & $-36.10 \mathrm{kcal} / \mathrm{mol}$ \\
\hline 61 & >cel-mir-233 Ml0000308 & $-38.92 \mathrm{kcal} / \mathrm{mol}$ & $8.53 \%$ & 4.73 & $-37.40 \mathrm{kcal} / \mathrm{mol}$ & $-37.40 \mathrm{kcal} / \mathrm{mol}$ \\
\hline 62 & >cel-mir-234 MI0000309 & $-27.04 \mathrm{kcal} / \mathrm{mol}$ & $8.26 \%$ & 4.88 & $-25.50 \mathrm{kcal} / \mathrm{mol}$ & $-25.50 \mathrm{kcal} / \mathrm{mol}$ \\
\hline 63 & >cel-mir-235 MI0000310 & $-31.29 \mathrm{kcal} / \mathrm{mol}$ & $1.76 \%$ & 13.20 & $-27.50 \mathrm{kcal} / \mathrm{mol}$ & $-28.80 \mathrm{kcal} / \mathrm{mol}$ \\
\hline 64 & >cel-mir-236 MI0000311 & $-38.76 \mathrm{kcal} / \mathrm{mol}$ & $3.55 \%$ & 7.62 & $-36.70 \mathrm{kcal} / \mathrm{mol}$ & $-36.70 \mathrm{kcal} / \mathrm{mol}$ \\
\hline 65 & >cel-mir-237 MI0000312 & $-37.15 \mathrm{kcal} / \mathrm{mol}$ & $3.57 \%$ & 6.10 & $-31.50 \mathrm{kcal} / \mathrm{mol}$ & $-35.10 \mathrm{kcal} / \mathrm{mol}$ \\
\hline 66 & >cel-mir-238 MI0000313 & $-39.38 \mathrm{kcal} / \mathrm{mol}$ & $10.60 \%$ & 6.22 & $-37.70 \mathrm{kcal} / \mathrm{mol}$ & $-38.00 \mathrm{kcal} / \mathrm{mol}$ \\
\hline 67 & >cel-mir-239a MI0000314 & $-33.64 \mathrm{kcal} / \mathrm{mol}$ & $13.28 \%$ & 4.78 & $-32.40 \mathrm{kcal} / \mathrm{mol}$ & $-32.40 \mathrm{kcal} / \mathrm{mol}$ \\
\hline 68 & >cel-mir-239b MI0000315 & $-38.52 \mathrm{kcal} / \mathrm{mol}$ & $31.16 \%$ & 2.88 & $-37.80 \mathrm{kcal} / \mathrm{mol}$ & $-37.80 \mathrm{kcal} / \mathrm{mol}$ \\
\hline 69 & >cel-mir-240 Ml0000316 & $-22.86 \mathrm{kcal} / \mathrm{mol}$ & $5.74 \%$ & 7.14 & $-20.90 \mathrm{kcal} / \mathrm{mol}$ & $-21.10 \mathrm{kcal} / \mathrm{mol}$ \\
\hline 70 & >cel-mir-241 MI0000317 & $-34.89 \mathrm{kcal} / \mathrm{mol}$ & $2.88 \%$ & 6.77 & $-32.20 \mathrm{kcal} / \mathrm{mol}$ & $-32.70 \mathrm{kcal} / \mathrm{mol}$ \\
\hline 71 & >cel-mir-244 Ml0000320 & $-36.99 \mathrm{kcal} / \mathrm{mol}$ & $20.12 \%$ & 4.55 & $-36.00 \mathrm{kcal} / \mathrm{mol}$ & $-36.00 \mathrm{kcal} / \mathrm{mol}$ \\
\hline 72 & >cel-mir-245 MI0000321 & $-31.62 \mathrm{kcal} / \mathrm{mol}$ & $16.36 \%$ & 8.64 & $-27.00 \mathrm{kcal} / \mathrm{mol}$ & $-30.50 \mathrm{kcal} / \mathrm{mol}$ \\
\hline 73 & >cel-mir-246 Ml0000322 & $-24.77 \mathrm{kcal} / \mathrm{mol}$ & $12.66 \%$ & 6.57 & $-23.50 \mathrm{kcal} / \mathrm{mol}$ & $-23.50 \mathrm{kcal} / \mathrm{mol}$ \\
\hline 74 & >cel-mir-247 MI0000323 & $-37.58 \mathrm{kcal} / \mathrm{mol}$ & $4.76 \%$ & 8.19 & $-35.70 \mathrm{kcal} / \mathrm{mol}$ & $-35.70 \mathrm{kcal} / \mathrm{mol}$ \\
\hline 75 & >cel-mir-250 MI0000326 & $-39.30 \mathrm{kcal} / \mathrm{mol}$ & $14.37 \%$ & 2.84 & $-38.50 \mathrm{kcal} / \mathrm{mol}$ & $-38.10 \mathrm{kcal} / \mathrm{mol}$ \\
\hline 76 & >cel-mir-253 MI0000329 & $-47.25 \mathrm{kcal} / \mathrm{mol}$ & $5.85 \%$ & 5.22 & $-45.30 \mathrm{kcal} / \mathrm{mol}$ & $-45.50 \mathrm{kcal} / \mathrm{mol}$ \\
\hline 77 & >cel-mir-255 MI0000331 & $-28.55 \mathrm{kcal} / \mathrm{mol}$ & $9.54 \%$ & 10.17 & $-24.10 \mathrm{kcal} / \mathrm{mol}$ & $-27.10 \mathrm{kcal} / \mathrm{mol}$ \\
\hline 78 & >cel-mir-259 MI0000336 & $-36.67 \mathrm{kcal} / \mathrm{mol}$ & $17.72 \%$ & 5.05 & $-35.60 \mathrm{kcal} / \mathrm{mol}$ & $-35.60 \mathrm{kcal} / \mathrm{mol}$ \\
\hline 79 & >cel-mir-355 Ml0000754 & $-37.24 \mathrm{kcal} / \mathrm{mol}$ & $2.26 \%$ & 9.21 & $-34.10 \mathrm{kcal} / \mathrm{mol}$ & $-34.90 \mathrm{kcal} / \mathrm{mol}$ \\
\hline
\end{tabular}


Citation: Dubey A, Agarwal SP, Yadav N, Kumar R (2016) Analysis of Caenorhabditis elegans via Bioinformatics Approaches Basis on their Precursors Statistics Values . Next Generat Sequenc \& Applic 3: 126. doi:10.4172/2469-9853.1000126

Page 4 of 5

\begin{tabular}{|c|c|c|c|c|c|c|}
\hline 80 & >cel-mir-356b MI0019158 & $-20.09 \mathrm{kcal} / \mathrm{mol}$ & $6.45 \%$ & 6.52 & $-15.30 \mathrm{kcal} / \mathrm{mol}$ & $-18.40 \mathrm{kcal} / \mathrm{mol}$ \\
\hline 81 & >cel-mir-358 MI0000757 & $-36.09 \mathrm{kcal} / \mathrm{mol}$ & $1.76 \%$ & 9.90 & $-33.60 \mathrm{kcal} / \mathrm{mol}$ & $-33.60 \mathrm{kcal} / \mathrm{mol}$ \\
\hline 82 & >cel-mir-392 MI0000819 & $-38.44 \mathrm{kcal} / \mathrm{mol}$ & $35.39 \%$ & 3.11 & $-37.80 \mathrm{kcal} / \mathrm{mol}$ & $-37.80 \mathrm{kcal} / \mathrm{mol}$ \\
\hline 83 & >cel-mir-784 MI0005184 & $-28.76 \mathrm{kcal} / \mathrm{mol}$ & $9.34 \%$ & 4.10 & $-24.50 \mathrm{kcal} / \mathrm{mol}$ & $-27.30 \mathrm{kcal} / \mathrm{mol}$ \\
\hline 84 & >cel-mir-786 MI0005186 & $-36.92 \mathrm{kcal} / \mathrm{mol}$ & $7.28 \%$ & 6.03 & $-35.30 \mathrm{kcal} / \mathrm{mol}$ & $-35.30 \mathrm{kcal} / \mathrm{mol}$ \\
\hline 85 & >cel-mir-787 MI0005187 & $-39.99 \mathrm{kcal} / \mathrm{mol}$ & $32.58 \%$ & 3.58 & $-39.30 \mathrm{kcal} / \mathrm{mol}$ & $-39.30 \mathrm{kcal} / \mathrm{mol}$ \\
\hline 86 & >cel-mir-788 MI0005188 & $-33.38 \mathrm{kcal} / \mathrm{mol}$ & $33.34 \%$ & 1.90 & $-32.70 \mathrm{kcal} / \mathrm{mol}$ & $-32.70 \mathrm{kcal} / \mathrm{mol}$ \\
\hline 87 & $\begin{array}{l}\text { >cel-mir-789-2 } \\
\text { MI0005190 }\end{array}$ & $-64.03 \mathrm{kcal} / \mathrm{mol}$ & $7.10 \%$ & 5.04 & $-60.50 \mathrm{kcal} / \mathrm{mol}$ & $-62.40 \mathrm{kcal} / \mathrm{mol}$ \\
\hline 88 & >cel-mir-790 MI0005191 & $-34.37 \mathrm{kcal} / \mathrm{mol}$ & $20.68 \%$ & 2.47 & $-33.30 \mathrm{kcal} / \mathrm{mol}$ & $-33.40 \mathrm{kcal} / \mathrm{mol}$ \\
\hline 89 & >cel-mir-791 MI0005192 & $-33.49 \mathrm{kcal} / \mathrm{mol}$ & $20.02 \%$ & 4.20 & $-32.50 \mathrm{kcal} / \mathrm{mol}$ & $-32.50 \mathrm{kcal} / \mathrm{mol}$ \\
\hline 90 & >cel-mir-794 MI0005195 & $-27.49 \mathrm{kcal} / \mathrm{mol}$ & $27.76 \%$ & 2.51 & $-26.70 \mathrm{kcal} / \mathrm{mol}$ & $-26.70 \mathrm{kcal} / \mathrm{mol}$ \\
\hline 91 & >cel-mir-795 MI0005196 & $-34.45 \mathrm{kcal} / \mathrm{mol}$ & $15.54 \%$ & 4.99 & $-33.30 \mathrm{kcal} / \mathrm{mol}$ & $-33.30 \mathrm{kcal} / \mathrm{mol}$ \\
\hline 92 & >cel-mir-797 MI0005198 & $-28.50 \mathrm{kcal} / \mathrm{mol}$ & $2.81 \%$ & 11.22 & $-26.10 \mathrm{kcal} / \mathrm{mol}$ & $-26.30 \mathrm{kcal} / \mathrm{mol}$ \\
\hline 93 & >cel-mir-800 MI0005201 & $-54.97 \mathrm{kcal} / \mathrm{mol}$ & $55.06 \%$ & 0.89 & $-54.60 \mathrm{kcal} / \mathrm{mol}$ & $-54.60 \mathrm{kcal} / \mathrm{mol}$ \\
\hline 94 & >cel-mir-1820 MI0007982 & $-40.02 \mathrm{kcal} / \mathrm{mol}$ & $22.60 \%$ & 5.32 & $-39.10 \mathrm{kcal} / \mathrm{mol}$ & $-39.10 \mathrm{kcal} / \mathrm{mol}$ \\
\hline 95 & >cel-mir-1821 MI0007983 & $-32.09 \mathrm{kcal} / \mathrm{mol}$ & $2.06 \%$ & 11.81 & $-27.20 \mathrm{kcal} / \mathrm{mol}$ & $-29.70 \mathrm{kcal} / \mathrm{mol}$ \\
\hline 96 & >cel-mir-1822 MI0007984 & $-32.27 \mathrm{kcal} / \mathrm{mol}$ & $17.50 \%$ & 4.79 & $-31.20 \mathrm{kcal} / \mathrm{mol}$ & $-31.20 \mathrm{kcal} / \mathrm{mol}$ \\
\hline 97 & >cel-mir-1823 MI0007985 & $-26.43 \mathrm{kcal} / \mathrm{mol}$ & $11.52 \%$ & 5.28 & $-22.70 \mathrm{kcal} / \mathrm{mol}$ & $-25.10 \mathrm{kcal} / \mathrm{mol}$ \\
\hline 98 & $\begin{array}{l}\text { >cel-mir-1829b } \\
\text { MI0008198 }\end{array}$ & $-21.74 \mathrm{kcal} / \mathrm{mol}$ & $67.49 \%$ & 1.00 & $-21.50 \mathrm{kcal} / \mathrm{mol}$ & $-21.50 \mathrm{kcal} / \mathrm{mol}$ \\
\hline 99 & $\begin{array}{l}\text { >cel-mir-1829c } \\
\text { MI0008199 }\end{array}$ & $-20.35 \mathrm{kcal} / \mathrm{mol}$ & $56.50 \%$ & 1.38 & $-20.00 \mathrm{kcal} / \mathrm{mol}$ & $-20.00 \mathrm{kcal} / \mathrm{mol}$ \\
\hline 100 & >cel-mir-1830 MI0008200 & $-35.96 \mathrm{kcal} / \mathrm{mol}$ & $34.49 \%$ & 3.70 & $-35.30 \mathrm{kcal} / \mathrm{mol}$ & $-35.30 \mathrm{kcal} / \mathrm{mol}$ \\
\hline 101 & $\begin{array}{l}\text { >cel-mir-1832a } \\
\text { MI0008202 }\end{array}$ & $-36.86 \mathrm{kcal} / \mathrm{mol}$ & $11.07 \%$ & 3.56 & $-35.50 \mathrm{kcal} / \mathrm{mol}$ & $-35.50 \mathrm{kcal} / \mathrm{mol}$ \\
\hline 102 & $\begin{array}{l}>\text { cel-mir-1832b } \\
\text { MI0010967 }\end{array}$ & $-44.60 \mathrm{kcal} / \mathrm{mol}$ & $32.19 \%$ & 2.79 & $-43.60 \mathrm{kcal} / \mathrm{mol}$ & $-43.90 \mathrm{kcal} / \mathrm{mol}$ \\
\hline 103 & $\begin{array}{l}\text { >cel-mir-2208a } \\
\text { MI0010956 }\end{array}$ & $-24.02 \mathrm{kcal} / \mathrm{mol}$ & $36.44 \%$ & 1.69 & $-23.40 \mathrm{kcal} / \mathrm{mol}$ & $-23.40 \mathrm{kcal} / \mathrm{mol}$ \\
\hline 104 & $\begin{array}{l}\text { >cel-mir-2208b } \\
\text { MI0010957 }\end{array}$ & $-27.27 \mathrm{kcal} / \mathrm{mol}$ & $33.97 \%$ & 1.94 & $-26.60 \mathrm{kcal} / \mathrm{mol}$ & $-26.60 \mathrm{kcal} / \mathrm{mol}$ \\
\hline 105 & >cel-mir-2221 MI0010974 & $-43.52 \mathrm{kcal} / \mathrm{mol}$ & $11.81 \%$ & 7.16 & $-42.20 \mathrm{kcal} / \mathrm{mol}$ & $-42.20 \mathrm{kcal} / \mathrm{mol}$ \\
\hline 106 & >cel-mir-4805 MI0017535 & $-49.11 \mathrm{kcal} / \mathrm{mol}$ & $16.52 \%$ & 8.53 & $-48.00 \mathrm{kcal} / \mathrm{moli}$ & $-48.00 \mathrm{kcal} / \mathrm{mol}$ \\
\hline 107 & >cel-mir-4813 MI0017543 & $-39.96 \mathrm{kcal} / \mathrm{mol}$ & $47.56 \%$ & 1.24 & $-39.50 \mathrm{kcal} / \mathrm{mol}$ & $-39.50 \mathrm{kcal} / \mathrm{mol}$ \\
\hline 108 & >cel-mir-4814 MI0017544 & $-66.59 \mathrm{kcal} / \mathrm{mol}$ & $38.34 \%$ & 2.76 & $-66.00 \mathrm{kcal} / \mathrm{mol}$ & $-66.00 \mathrm{kcal} / \mathrm{mol}$ \\
\hline 109 & >cel-mir-4816 MI0017546 & $-24.54 \mathrm{kcal} / \mathrm{mol}$ & $35.29 \%$ & 2.53 & $-23.90 \mathrm{kcal} / \mathrm{mol}$ & $-23.90 \mathrm{kcal} / \mathrm{mol}$ \\
\hline 110 & >cel-mir-5545 MI0019066 & $-64.97 \mathrm{kcal} / \mathrm{mol}$ & $46.98 \%$ & 2.35 & $-64.50 \mathrm{kcal} / \mathrm{mol}$ & $-64.50 \mathrm{kcal} / \mathrm{mol}$ \\
\hline 111 & $\begin{array}{l}\text { >cel-mir-5592-1 } \\
\text { MI0019153 }\end{array}$ & $-40.09 \mathrm{kcal} / \mathrm{mol}$ & $27.56 \%$ & 2.99 & $-39.30 \mathrm{kcal} / \mathrm{mol}$ & $-39.30 \mathrm{kcal} / \mathrm{mol}$ \\
\hline
\end{tabular}


Citation: Dubey A, Agarwal SP, Yadav N, Kumar R (2016) Analysis of Caenorhabditis elegans via Bioinformatics Approaches Basis on their Precursors Statistics Values . Next Generat Sequenc \& Applic 3: 126. doi:10.4172/2469-9853.1000126

Page 5 of 5

\begin{tabular}{|l|l|l|l|l|l|l|}
\hline 112 & $\begin{array}{l}>\text { cel-mir-5592-2 } \\
\text { Ml0019154 }\end{array}$ & $-41.50 \mathrm{kcal} / \mathrm{mol}$ & $32.17 \%$ & 3.28 & $-40.80 \mathrm{kcal} / \mathrm{mol}$ & $-40.80 \mathrm{kcal} / \mathrm{mol}$ \\
\hline 113 & $>$ cel-mir-5594 Ml0019157 & $-26.98 \mathrm{kcal} / \mathrm{mol}$ & $14.80 \%$ & 3.10 & $-25.80 \mathrm{kcal} / \mathrm{mol}$ & $-25.80 \mathrm{kcal} / \mathrm{mol}$ \\
\hline
\end{tabular}

Table 1: This table explains that miRBase precursors of $C$. elegans. These sequences pass from RNA fold web server for thermodynamics analysis with minimum free energy

\section{Results and Discussion}

Here we have analyzed optimal minimum free energy (MFE) of miRBase precursors of C.elegans. Present study is exclusively based on in silico. Firstly we retrives precursor sequences from miRBase and then this sequence is submitted in RNA fold web server for minimum free energy values.

The most common software programs, employed to predict the secondary RNA structures by MFE algorithms, make use of the socalled nearest-neighbor energy model. This model uses free energy rules based on empirical thermodynamic parameters and computes the overall stability of an RNA structure by adding independent contributions of local free energy interactions due to adjacent base pairs and loop regions. In sequences with homogeneous nucleotide arrangements and compositions, the additive and independent nature of the local free energy contributions suggests a linear relationship between computed MFE and sequence length. Normalization by length, obtained by dividing MFE by the number of nucleotides, was introduced to exploit this linear relationship to directly compare the minimum free energies of RNAs of various lengths $[14,15]$.

In Table 1 we have predicted Free energy of thermodynamics, Frequency of MFE structure, Ensemble diversity, Minimum free energy of centeriod secondary structure. The minimum free energy (MFE) of ribonucleic acids (RNAs) increases at an apparent linear rate with sequence length. Simple indices, obtained by dividing the MFE by the number of nucleotides, have been used for a direct comparison of the folding stability of RNAs of various sizes.

\section{Conclusion}

In this study entitled: Analysis of $C$. elegans via bioinformatics approaches basis on their precursors statistics values, analyze the statistical values of miRNA and their precursors. For computational analysis of miRNA we always predict the MFE values from precursor sequences which is already experimentally identified and this precursor sequences retrieves from miRBase, for miRNA targeted genes and other analysis also. This table explains all precursors and miRNAs mainly important values, these values always used in noncoding RNA analysis via the system biology. Our computational findings may be useful for researchers.

\section{Acknowledgements}

The corresponding author wish to thanks Er. NEELESH YADAV, Scientist and Coordinator, BioinformaticsCentre, Forest Research
Institute, Dehradun, INDIA for providing valuable scientific platform . I am also thankful to DBT center for providing necessary facilities.

\section{References}

1. Dubey A, Agrawal SP, Yadav N (2015) Evidences for big roles of miRNAs from Pristionchus pacificus to human targeted genes via bioinformatics approaches. American Journal of Biological, Chemical and Pharmaceutical Sciences 3: 1-15.

2. Saxena VL, Dwivedi A (2013) In silico identification of miRNAs and their target prediction from Japanese encephalitis. Journal of Bioinformatics and Sequence Analysis 5: 25-33.

3. Dubey A, Shanker U, Kalra SS, Yadav N (2013) Viral micro RNA analysis via the bioinformatics approaches basis on their Statistics values. American Journal of Biological, Chemical and Pharmaceutical Sciences 1: $42-66$.

4. Lee RC, Feinbaum RL, Ambros V (1993) The C. elegansheterochronic gene lin- 4 encodes small RNAs with antisense complementarity to lin-14. Cell 75: 843-854.

5. Lagos-Quintana $\mathrm{M}$, Rauhut $\mathrm{R}$, Lendeckel W, Tuschl $\mathrm{T}$ (2001) Identification of novel genes coding for small expressed RNAs. Science 294: 853-858.

6. Lau NC, Lim LP, Weinstein EG, Bartel DP (2001) An abundant class of tiny RNAs with probable regulatory roles in Caenorhabditiselegans. Science 294: 858-862.

7. Llave C, Kasschau KD, Rector MA, Carrington JC (2002) Endogenous and silencing-associated small RNAs in plants. Plant Cell 14: 1605-1619.

8. Reinhart BJ, Weinstein EG, Rhoades MW, Bartel B, Bartel DP(2002) MicroRNAs in plants .Genes Dev 16: 1616-1626.

9. Hutvagner G, Zamore PD (2002) RNAi: nature abhors a doublestrand. Curr Opin Genet Dev 12: 225-232.

10. Schauer SE, Jacobsen SE, Meinke DW, Ray A (2002) DICER-LIKE1: blind men and elephants in Arabidopsis development. Trends Plant Sci 7: 487-491.

11. Reinhart BJ, Slack FJ, Basson M, Pasquinelli AE, Bettinger JC, et al. (2000) The 21-nucleotide let-7 RNA regulates developmental timing in Caenorhabditiselegans. Nature 403: 901-906.

12. Ambros V, Bartel B, Bartel DP, Burge CB, Carrington JC, et al. (2003) A uniform system for microRNA annotation.RNA 9: 277- 279.

13. Kibbe WA (2007) An online oligonucleotide properties calculator.Nucleic Acids Research 35: W43-46.

14. Mathews DH, Sabina J, Zuker M, Turner DH (1999) Expanded sequence dependence of thermodynamic parameters improves prediction of RNA secondary structure. J Mol Biol 288: 911-940.

15. Mathews DH, Disney MD, Childs JL, Schroeder SJ, Zuker M, et al. (2004) Incorporating chemical modification constraints into a dynamic programming algorithm for prediction of RNA secondary structure. Proc Natl Acad Sci U S A 101: 7287-7292. 